\title{
Energetic flares on RS CVn stars
}

\author{
J.G. Doyle* \\ P.B. Byrne* \\ G.H.J. van den Oord ${ }^{+}$
}

*Armagh Observatory, Armagh BT61 9DG, N. Ireland

+Dept. of Plysics \& Astronomy, University of Glasgow, Glasgow G12 8QQ,

Scotland

\begin{abstract}
Ultravialet spectroscopic observations of the RS CVn star II Peg in February 1983 show evidence for flare activity in greatly enhanced chromospheric and transition region emission lines. The total radiative losses from the chromosphere and transition region (i.e. the temperature interval $4.0 \leq \log T_{\mathrm{e}} \leq 5.4$ ) during the flare is $3.110^{35} \mathrm{erg}$. Over the whole atmosphere (i.e. the temperature interval $4.0 \leq \log T_{\mathrm{e}} \leq 8.0$ ), we estimate total radiative losses of $2.410^{36} \mathrm{erg}$, (cxcluding hydrogen line radiation). At flare peak, the flare radiated $1.510^{32} \mathrm{erg} \mathrm{s}^{-1}$. Adopting a two-ribbon flare model, where the filament is located between the two stars of the system, we can have $20^{39}\left(l / R_{\odot}\right)$ erg of magnetic energy available, where $l$ is the filament length and we have talen a magnetic field strength of $1000 \mathrm{G}$. Therefore, only a small fraction of this magnetic energy need be converted into heating of the flare plasma.
\end{abstract}

\section{Introduction \& Observational Data}

II Peg (=HD 224085) is a single-line spectroscopic binary of spectral type K2 IV-V with an orbital period of approximately 6.7 days. It is photometrically variable showing a wave-like variation, of the type usually interpreted as due to cool surface spots. Flares on RS CVn stars are quite often detected in the radio regime. These radio flares can last for up to days and involve characteristic radius up to several stellar radii. Faster ultraviolet and optical emission line and microwave radio flares have also been recorded lasting, typically, several hours. Here, we discuss an IUE observation of a large flare observed on this star in February 1983. This flare was 
first detected in an IUE exposure at 23:54 UT on Feb. 1 and lasted until at least 05:36 UT on Feb. 2. Three separate IUE SWP exposures were obtained during this time interval.

Line fluxes for the prominent emission lines were derived by least-squares gaussian fits. The FWIIM of the fitted lines were consistent with the instrumental width of $\approx 5 . \AA$ for low resolution SWP spectra. The derived line fluxes at Earth were reduced to surface fluxes at the star using the conversion factor, $2.310^{17}$. All of the prominent lines were considerably enhanced during the flares. The cuhancement factors range up to 9 for NV (1238/42).

\section{Results}

In order to discuss the properties of the flare plasma we have isolated the radiation of the flare from the background quiescent stellar flux by subtracting the mean quiescent spectrum from each of the flare spectra.

The radiative losses per second per unit surface area over the temperature interval $4.3 \leq \log T_{e} \leq 5.4$ can be calculated by multiplying the derived EM curve by the radiative loss function (see Byrne et al 1087 for details). The total energy radiated by the flares' transition region is derived by multiplying by the stellar surface area, and assuming that we can integrate the losses over the time interval from the beginning of the first sjectrum on which we detected the flare to the end of the last flare spectrum using a simple linear interpolation. This procedure yields a total radiative energy over the temperature range $4.3 \leq \log T_{e} \leq 5.4$ of a.t least $1.910^{35} \mathrm{crg}$. It should be stressed that this is an under-estimate since we do not in fact know when the flare began or ended. The error in our knowledge of the start time is likely to be the more serious error since the three consecutive flare spectra exhibit a monotonic decay which can be extrapolated with reasonable confilence.

The above values for the radiative losses are only for the upper chromosphere and transition region. Recently, it has been shown that, for a variety of solar plasmas, the total radiated power output, exclusive of hydrogen line radiation, may be estimated even if one hnows the intensity of only one spectral line, e.g. C IV 1548.4 (Bruner \& McWhirter 1088). If we assume that this similarity extends to RS CVn stars then the observed C IV $1548 \AA$ flux yields total radiative losses of $2.410^{36} \mathrm{erg}$. These estimates for the total power output can be considered as 
- a lower limit to the required mechanical input to the atmosphere. This radiative energy budget is a factor of $10^{4}$ larger than found for solar two-ribbon flares. Below we discuss if a two-ribbon flare situated between the two stars could explain this large energy budget.

\section{3 - Discussion}

\section{1 flare energy implications}

A two-ribbon flare is associated with the presence of a filament in an active region which becomes unstable (van Tend \& Kuperus 1978). The filament can be regarded as a current wire which has its own magnetic field associated with it. This magnetic field cannot penetrate the photosphere because of the large inertia of the photospheric plasma. As a result, surface currents will be generated and consequently the magnetic field in the corona will be modified.

Neglecting gravity, the force on the filament consists of the Lorentz force by the background magnetic field and by a surface current magnetic field. The shearing motions of the photospheric footpoints result in an increase of the current in the filament. It can be shown that as the filament moves upward along an equilibrium curve, titen at some critical height force equilibrium is lost and a two-ribbon flare results. We adopt this model to the situation that the filament is located in a binary system (van den Oord 1988). The presence of a second component results in the increase of the stored energy since also surface currents will be generated on this secondary. Whether an observable flare will occur depends on two factors. First, enough energy must be stored in the pre-flare configuration and second, there must be a possibility for an instability. The total energy available for the flare is the energy stored in the preflare configuration at the moment that the instability occurs.

It can be shown that the maximal storage of energy is obtained when the filament is located between both binary components. The total energy stored in the system for that situation is

$$
W=410^{37}\left(\frac{l}{R_{\odot}}\right)\left(\frac{a}{R_{\odot}}\right)^{2}\left(\frac{B_{\text {surf }}}{1000 G}\right)^{2} \quad \text { erg }
$$

with $l$ the length of the filament, $a$ the binary separation, $R_{\odot}$ the solar radius and $B_{\text {surf }}$ the surface magnetic field strength of the active component in the binary. 
Although the orlital parameters of II Peg are poorly known, this expression shows that sufficient energy can be stored to explain the flare discussed in this paper. The binary nature of the system allows storage of a factor $\left(1.0 a / R_{*}\right)^{2}$ more energy in the filament/surface current system than is present in the active region background ficld. For example, if the flare occurred on a single star with a comparable background field, the stored energy would therefore be a factor of $\left(1.6 a / R_{*}\right)^{2}$ lower resulting in

$$
W_{\text {single }}=1.010^{37}\left(\frac{l}{R_{\odot}}\right)\left(\frac{R_{*}}{R_{\odot}}\right)^{2}\left(\frac{B_{\text {sur }}}{1000 G}\right)^{2} \text { erg }
$$

Inserting the values for $l, a, R_{*}$ and $B_{\text {sur } f}$ in general results in an energy estimate which is sufficient to power the most energetic flares. It is however important to note that only a small fraction of the initially stored energy is converted to radiation. In some solar flares, only 0.003 of the magnetic energy released actually goes into heating of the plasma (this fraction would obviously vary from flare-toflire). If we were to assume that a similar fraction of the magnetic energy went into heating the flare plasma for the 2 Feb. flare, then we would required a total encergy storage of the order of $10^{39} \mathrm{erg}$. Taking the stellar radius $R_{*}=2.8 R_{\odot}$, a binary separation of $a=4.9 R_{\odot}$ and the inclination to be $\approx 90^{\circ}$ and a magnetic field strength of $1000 \mathrm{G}$, then we have from equ. (1) $W \approx 110^{39}\left(l / R_{\odot}\right)$ erg and from equu. (2) $W_{\text {single }} \approx 110^{38}\left(l / R_{\odot}\right)$ erg. These numbers indicate that the binary nature of the system indeed plays an important role in the storage of the flare energy in the pre-flare phase.

\section{References}

[1] Bruner, M.E. \& McWlirter, R.W.P.: 1988, Astrophys. J 326,1002

[2] Byrne, P.B., Doyle, J.G., Brown, A., Linsky, J.L., rodono, M: 1987, Astron. Astrophys. 180,172

[3] vau den Oord, G.II.J.: 1088, Astron. Astrophys. (in press)

[-1] van Tend, W. \& Kuperus, M.: 1978, Solar Phys. 58,115 